\title{
Impact of cytomegalovirus reactivation on clinical outcomes in immunocompetent critically ill patients: protocol for a systematic review and meta-analysis
}

Philippe Lachance ${ }^{1 *} \mathbb{D}$, Justin Chen ${ }^{2}$, Robin Featherstone ${ }^{3}$ and Wendy Sligl $\left.\right|^{1,2}$

\begin{abstract}
Background: Cytomegalovirus (CMV) reactivation in critically ill patients is a well-recognized phenomenon with an incidence as high as $71 \%$. A number of studies have investigated the association between CMV reactivation and outcomes in critically ill patients with conflicting results. We propose to conduct a systematic review and metaanalysis to determine the impact of CMV reactivation on patient-centered outcomes and measures of health resource utilization in immunocompetent critically ill patients.

Methods: In consultation with a research librarian, a search strategy will be developed and electronic databases (i.e., Ovid MEDLINE, Ovid EMBASE, and the Cochrane Library including the Cochrane Database of Systematic Reviews, the Cochrane Central Register of Controlled Trials (CENTRAL)) will be searched for original studies. Selected grey literature sources will be hand-searched. Search themes will include cytomegalovirus, intensive care unit, and sepsis. Citation screening, selection, quality assessment, and data abstraction will be performed in duplicate. Pooled effect estimates of the impact of CMV reactivation on selected patient-centered outcomes and measures of health resource utilization will be described.

Discussion: This systematic review aims to explore the impact of CMV reactivation on patient-centered outcomes and health resource utilization in immunocompetent critically ill patients. Our results will help to better define the burden of disease associated with CMV reactivation. Indeed, evidence to date suggests increased mortality in this patient population. However, the relationship between CMV reactivation and health resource utilization remains less clear. Based on our results, future study on the impact of CMV treatment or prophylaxis on outcomes (including those other than mortality) may be warranted.
\end{abstract}

Systematic review registration: PROSPERO CRD42016035446

Keywords: Cytomegalovirus, Intensive care unit, Meta-analysis, Systematic review, Protocol

\section{Background}

It is estimated that 40 to $100 \%$ of immunocompetent adults are cytomegalovirus (CMV) seropositive globally [1,2]. In Canada, seroprevalence ranges between 60 and $80 \%$ [2]. Most primary infections occur in childhood and are subclinical or present with non-specific

\footnotetext{
* Correspondence: plachanc@ualberta.ca

${ }^{1}$ Division of Critical Care Medicine, Faculty of Medicine and Dentistry, University of Alberta, 2-124 Clinical Sciences Building, 8440 - 112th Street, Edmonton, Alberta T6G 2B7, Canada

Full list of author information is available at the end of the article
}

symptoms. CMV subsequently remains latent in monocytes and macrophages $[3,4]$. This state of latency allows CMV to reactivate when host defenses become compromised, such as in critical illness. CMV reactivation in critically ill patients is well-recognized with as high as $71 \%$ incidence [5]. The consequences of CMV reactivation in immunocompromised patient populations, such as solid organ transplantation, have been well described [6]. However, the clinical significance in immunocompetent patients remains controversial. Some postulate viral pathogenesis by direct cytopathic effect (tissue-invasive 
disease) [5, 7-9], by an over-response of the immune system $[5,10]$ or by inactivation of host defenses allowing opportunistic pathogens to establish infection $[5,11$, 12]. Others have suggested that CMV reactivation is only a marker of illness severity [5].

Since the 1990s, a number of studies have investigated the association between CMV reactivation and outcomes in critically ill patients. In 1990, Domart et al. examined patients with mediastinitis following cardiac surgery who were CMV infected, defined by blood and/or urine viral cultures. They showed a significant increase in mortality and hospital length of stay compared with CMVuninfected patients [13]. Thereafter, other studies have also reported increased mortality $[14,15]$, increased duration of mechanical ventilation $[11,12]$, increased length of intensive care unit (ICU) stay [16, 17], and increased incidence of nosocomial infections [18]. Contrasting this data, Heininger et al. failed to demonstrate a difference in in-hospital mortality in patients with CMV DNAemia [19]. More recently, Frantzeskaki et al. came to the same conclusion [20].

With a growing number of studies examining the impact of CMV reactivation on outcomes and discrepancies in the available data, systematic reviews and meta-analyses have been previously undertaken. In 2009, Osawa et al. conducted the first systematic review on the subject, which included 13 studies. Four studies reported data on duration of mechanical ventilation-all of which showed a statistically significant negative effect of CMV reactivation on this outcome. No pooled estimate was available, as they did not perform a meta-analysis. All but two of the included studies reporting death showed no difference between CMV positive and negative patients and mortality [21]. Conversely, Kalil et al. published a meta-analysis the same year including eight studies and 633 patients showing a twofold increase in the odds ratio of death with CMV infection. There was however no data on other clinical outcomes [22]. These authors updated their results after Heininger et al. published the study mentioned above showing no difference in mortality [19]. The effect of CMV infection on mortality remained significant [23]. Finally, Coisel et al. performed a prospective study on the prognosis of CMV-infected mechanically ventilated patients in which they included a meta-analysis demonstrating a positive association between CMV antigenemia and mortality [24]. Since the publication of the last meta-analysis, at least four additional studies have been published on this topic with varying results [20, 25-27].

Considering the availability of new evidence and the absence of meta-analyses examining important outcomes such as mechanical ventilation duration, ICU length of stay, or incidence of nosocomial infection, we propose to conduct a systematic review and meta-analysis to determine the impact of CMV reactivation on various clinical outcomes in immunocompetent critically ill patients.

\section{Objectives}

The aim of our systematic review is to determine the impact of CMV reactivation (defined by either pp65 CMV antigenemia or blood/plasma CMV DNAemia detected by quantitative nucleic acid testing [NAT]) compared to no reactivation on patient-centered outcomes (including mortality, duration of mechanical ventilation, nosocomial infection) or health services utilization (ICU length of stay, hospital length of stay) in immunocompetent critically ill patients.

\section{Methods}

\section{Study design}

A systematic review will be performed using guidelines from The Cochrane Collaboration and Center for Reviews and Dissemination and described according to PRISMA-P guidelines (available at: http://www.systematicreviewsjournal.com/content/4/1/1) (see Additional file 1) $[28]$.

\section{Study registration}

In accordance with PRISMA-P guidelines, our systematic review will be registered with the International Prospective Register of Systematic Reviews (PROSPERO) (www.crd.york.ac.uk/prospero; registration number CRD42016035446).

\section{Criteria for considering studies for this review}

Inclusion criteria:

1. Population: We will include studies of adults [ $>18$ years of age] who are immunocompetent [i.e., we will specifically exclude solid organ or bone marrow transplant patients, those with advanced HIV/AIDS, or those receiving cytotoxic therapies] admitted to any type of ICU for any cause. Patients with documented CMV tissue invasive disease will also be excluded.

2. Intervention: Critically ill patients with $\mathrm{CMV}$ reactivation, defined by either pp65 CMV antigenemia or blood/plasma DNAemia, will be compared to those without reactivation.

3. Outcomes: We will examine the impact of CMV reactivation on at least one patient-centered outcome or measure of health resource utilization.

4. Design: We will include observational studies (prospective and retrospective) as well as randomized trials. We will exclude case reports and case series. 


\section{Exclusion criteria}

Studies will be excluded if they do not fulfill all of the inclusion criteria; if they are published in a language other than English or French, use serology to define CMV reactivation, or include immunocompromised patients (as defined above).

\section{Search methods for identification of studies}

PROSPERO (http://www.crd.york.ac.uk/prospero) was searched for any registered systematic reviews on this topic (November 30, 2015).

The search strategy was developed in consultation with an expert librarian/information specialist at the Alberta Research Centre for Health Evidence (ARCHE) at the University of Alberta and has undergone subsequent peer-review by a second specialized librarian using the Peer Review of Electronic Search Strategies checklist [29]. The information specialist will search electronic databases: Ovid MEDLINE, Ovid EMBASE, and the Cochrane Library including the Cochrane Database of Systematic Reviews, the Cochrane Central Register of Controlled Trials (CENTRAL) for three domains: cytomegalovirus, intensive care unit, and sepsis. Database search results will be restricted to papers published in English or French language and published after 1990 for screening.

Appropriate truncation and wildcards will be used in the search to account for plurals and/or variations in the spelling of search terms (see Additional file 2 for example of the search strategy in Medline). Bibliographic records will be exported to EndNote X7 (Thomson Reuters, Philadelphia, Pennsylvania) for screening. Additional sources will be included in the search strategy. The cited and citing references of selected key studies will be searched for relevant articles. Grey literature sources will be searched. We will identify and search relevant conference proceedings from the past 2 years: Infectious Diseases Society of America (IDSA) IDWeek, Canadian Association for Clinical Medical Microbiology and Infectious Diseases-Association of Medical Microbiology and Infectious Diseases Canada (CACMIDAMMI) Annual Meeting, European Society of Clinical Microbiology and Infectious Diseases (ESCMID) Annual Congress, Society of Critical Care Medicine Annual Congress, International Symposium on Intensive Care and Emergency Medicine. We will also search the trial registry at www.clinicaltrials.gov for trials conducted during the past 2 years.

\section{Study selection}

Potentially eligible articles will be identified by two authors after independent review of the titles and abstracts of all articles identified by the search. The full text of all articles deemed potentially relevant will be independently reviewed, again by the two authors, for inclusion using pre-defined eligibility criteria. Any disagreements that arise will be resolved through discussion and/or arbitration by the senior author. Inter-rater agreement will be calculated.

\section{Data extraction}

Data will be abstracted from relevant studies using a standardized electronic data collection form (Additional file 3). Data extracted will include publication-related information, patient-related information (demographic characteristics and medical comorbidities, design, and quality assessments of the included studies, inclusion, and exclusion criteria), and the method of CMV detection. Patient-related outcomes and health service use will also be collected. This form will undergo pilot testing. Abstraction will be performed in duplicate by the same two authors. Any disagreements that arise will be resolved through discussion or arbitration by the senior author. The authors of the retrieved studies and/or documents will be contacted for further information as necessary.

Study methodological quality will be rated using the Newcastle-Ottawa Scale (NOS) [30] for observational studies and the Cochrane Collaboration's tool [31] for trials.

\section{Outcomes}

The primary outcome of our study will be mortality (however defined in the included studies). Secondary outcomes will be mechanical ventilation duration, nosocomial infections, need for renal replacement therapy, ICU length of stay, and hospital length of stay.

\section{Analysis}

Pooled effect estimates of the impact of CMV reactivation on patient-centered outcomes and health service use will be reported. We will assess and quantify statistical heterogeneity for each pooled summary estimate using Cochran's Q statistic and the $I^{2}$ statistic, respectively [32]. Pooled analysis will be performed using random effects models and reported as odds ratios with $95 \%$ confidence intervals for categorical variables and weighted mean differences with $95 \%$ confidence intervals for continuous variables, respectively. We expect to see heterogeneity as a result of different CMV detection methods, varied study designs, and due to the evolution in ICU care over time. To address this, we plan to perform a number of pre-defined sensitivity analyses according to the following variables: study design (observational vs. RCT), year of study (before or after 2005), and studies including only mechanically ventilated patients. Publication bias will be assessed using Egger's regression models and visualized using funnel plots [33]. All analyses will be performed using RevMan statistical software. 


\section{Expected limitations}

Based on screening of the literature, we expect some degree of heterogeneity in our study populations and in the frequency of CMV monitoring. The latter may affect our ability to detect CMV reactivation. Heterogeneity may limit the interpretation of our results.

Inter-laboratory CMV viral loads using laboratorydeveloped NATs can vary significantly-especially at low viral loads (from $2 \log _{10}$ copies/mL to $4.3 \log _{10}$ copies/ $\mathrm{mL}$ on the same specimen) and when testing was performed prior to the development of WHO International Calibration Standards in 2010 [34]. We expect that this will have minimal impact on our results as we will use any CMV antigenemia or DNAemia to define CMV reactivation.

\section{Discussion}

This systematic review and meta-analysis will explore the association between CMV reactivation, patientcentered outcomes, and health resource utilization in immunocompetent critically ill patients. Our results will help to better define the burden of disease associated with CMV reactivation. Indeed, evidence to date suggests increased mortality in this patient population. However, the relationship between CMV reactivation and health resource utilization is less clear. Based on our results, future study on the impact of CMV treatment or prophylaxis on outcomes (including those other than mortality) may be warranted.

\section{Additional files}

Additional file 1: Preferred Reporting Items for Systematic review and Meta-Analysis Protocols (PRISMA-P) 2015 checklist: recommended items to address in a systematic review protocol. (DOC $120 \mathrm{~kb}$ )

Additional file 2: Example of the search strategy in Medline. ( DOC $113 \mathrm{~kb}$ )

Additional file 3: Data to be collected. (DOC $99.7 \mathrm{~kb}$ )

\section{Abbreviations}

AIDS, acquired immunodeficiency syndrome; ARCHE, Alberta Research Centre for Health Evidence; CACMID-AMMI, Canadian Association for Clinical Medical Microbiology and Infectious Diseases-Association of Medical Microbiology and Infectious Diseases Canada; CENTRAL, Cochrane Central Register of Controlled Trials; CMV, cytomegalovirus; DNA, deoxyribonucleic acid; ESCMID, European Society of Clinical Microbiology and Infectious Diseases; HIV, human immunodeficiency virus; ICU, intensive care unit; IDSA, Infectious Diseases Society of America; NAT, nuclear acid testing; RCT, randomized control trial

\section{Acknowledgements}

We acknowledge Tara Landry for her peer review of the search strategy.

\section{Funding}

None.

\section{Authors' contributions}

PL conceived the study and drafted the manuscript. WS conceived the study, drafted the manuscript, and is the guarantor of the review. RF created the research strategy. JC reviewed the manuscript and provided his comment. All authors read and approved the final manuscript.

\section{Competing interests}

The authors declare that they have no competing interests.

\section{Author details}

${ }^{1}$ Division of Critical Care Medicine, Faculty of Medicine and Dentistry, University of Alberta, 2-124 Clinical Sciences Building, 8440 - 112th Street, Edmonton, Alberta T6G 2B7, Canada. ${ }^{2}$ Division of Infectious Diseases, Faculty of Medicine and Dentistry, University of Alberta, Edmonton, Canada. ${ }^{3}$ Alberta Research Centre for Health Evidence (ARCHE), Department of Pediatrics,

University of Alberta, Edmonton, Canada.

Received: 4 March 2016 Accepted: 18 July 2016

Published online: 28 July 2016

\section{References}

1. Krech U. Complement-fixing antibodies against cytomegalovirus in different parts of the world. Bull World Health Organ. 1973;49(1):103-6.

2. Cannon MJ, Schmid DS, Hyde TB. Review of cytomegalovirus seroprevalence and demographic characteristics associated with infection. Rev Med Virol. 2010;20(4):202-13.

3. Kondo K, Kaneshima H, Mocarski ES. Human cytomegalovirus latent infection of granulocyte-macrophage progenitors. Proc Natl Acad Sci U S A. 1994:91(25):11879-83.

4. Taylor-Wiedeman J, Sissons JG, Borysiewicz LK, Sinclair JH. Monocytes are a major site of persistence of human cytomegalovirus in peripheral blood mononuclear cells. J Gen Virol. 1991;72(Pt 9):2059-64.

5. Papazian L, Hraiech S, Lehingue S, Roch A, Chiche L, Wiramus S, et al. Cytomegalovirus reactivation in ICU patients. Intensive Care Med. 2016;42(1):28-37.

6. Requiao-Moura LR, deMatos AC, Pacheco-Silva A. Cytomegalovirus infection in renal transplantation: clinical aspects, management and the perspectives. Einstein (Sao Paulo). 2015;13(1):142-8

7. Papazian L, Fraisse A, Garbe L, Zandotti C, Thomas P, Saux P, et al. Cytomegalovirus. An unexpected cause of ventilator-associated pneumonia. Anesthesiology. 1996;84(2):280-7.

8. Papazian L, Thomas P, Bregeon F, Garbe L, Zandotti C, Saux P, et al. Openlung biopsy in patients with acute respiratory distress syndrome. Anesthesiology. 1998;88(4):935-44.

9. Barry SM, Johnson MA, Janossy G. Cytopathology or immunopathology? The puzzle of cytomegalovirus pneumonitis revisited. Bone Marrow Transplant. 2000;26(6):591-7.

10. Grundy JE, Shanley JD, Griffiths PD. Is cytomegalovirus interstitial pneumonitis in transplant recipients an immunopathological condition? Lancet. 1987;2(8566):996-9.

11. Chiche L, Forel JM, Roch A, Guervilly C, Pauly V, Allardet-Servent J, et al. Active cytomegalovirus infection is common in mechanically ventilated medical intensive care unit patients. Crit Care Med. 2009;37(6):1850-7.

12. Cook CH, Martin LC, Yenchar JK, Lahm MC, McGuinness B, Davies EA, et al. Occult herpes family viral infections are endemic in critically ill surgical patients. Crit Care Med. 2003;31(7):1923-9.

13. Domart Y, Trouillet IL, Fagon JY, Chastre J, Brun-Vezinet F, Gibert C. Incidence and morbidity of cytomegaloviral infection in patients with mediastinitis following cardiac surgery. Chest. 1990;97(1):18-22.

14. Limaye AP, Kirby KA, Rubenfeld GD, Leisenring WM, Bulger EM, Neff MJ, et al. Cytomegalovirus reactivation in critically ill immunocompetent patients. JAMA. 2008;300(4):413-22.

15. Lopez Roa P, Perez-Granda MJ, Munoz P, Catalan P, Alonso R, Sanchez-Perez $E$, et al. A prospective monitoring study of cytomegalovirus infection in non-immunosuppressed critical heart surgery patients. PLoS One. 2015; 10(6):e0129447.

16. Cook CH, Yenchar JK, Kraner TO, Davies EA, Ferguson RM. Occult herpes family viruses may increase mortality in critically ill surgical patients. Am J Surg. 1998;176(4):357-60.

17. Heininger A, Jahn G, Engel C, Notheisen T, Unertl K, Hamprecht K. Human cytomegalovirus infections in nonimmunosuppressed critically ill patients. Crit Care Med. 2001;29(3):541-7.

18. Jaber S, Chanques G, Borry J, Souche B, Verdier R, Perrigault PF, et al. Cytomegalovirus infection in critically ill patients: associated factors and consequences. Chest. 2005;127(1):233-41. 
19. Heininger A, Haeberle H, Fischer I, Beck R, Riessen R, Rohde F, et al. Cytomegalovirus reactivation and associated outcome of critically ill patients with severe sepsis. Crit Care. 2011;15(2):R77.

20. Frantzeskaki FG, Karampi ES, Kottaridi C, Alepaki M, Routsi C, Tzanela M, et al. Cytomegalovirus reactivation in a general, nonimmunosuppressed intensive care unit population: incidence, risk factors, associations with organ dysfunction, and inflammatory biomarkers. J Crit Care. 2015;30(2): 276-81.

21. Osawa R, Singh N. Cytomegalovirus infection in critically ill patients: a systematic review. Crit Care. 2009;13(3):R68.

22. Kalil AC, Florescu DF. Prevalence and mortality associated with cytomegalovirus infection in nonimmunosuppressed patients in the intensive care unit. Crit Care Med. 2009;37(8):2350-8.

23. Kalil AC, Florescu DF. Is cytomegalovirus reactivation increasing the mortality of patients with severe sepsis? Crit Care. 2011:15(2):138.

24. Coisel Y, Bousbia S, Forel JM, Hraiech S, Lascola B, Roch A, et al. Cytomegalovirus and herpes simplex virus effect on the prognosis of mechanically ventilated patients suspected to have ventilator-associated pneumonia. PLoS One. 2012;7(12):e51340.

25. Walton AH, Muenzer JT, Rasche D, Boomer JS, Sato B, Brownstein BH, et al. Reactivation of multiple viruses in patients with sepsis. PLoS One. 2014:9(2):e98819.

26. Lopez Roa P, Hill JA, Kirby KA, Leisenring WM, Huang ML, Santo TK, et al. Coreactivation of human herpesvirus 6 and cytomegalovirus is associated with worse clinical outcome in critically ill adults. Crit Care Med. 2015;43(7): 1415-22.

27. Ong DS, Klein Klouwenberg PM, Verduyn Lunel FM, Spitoni C, Frencken JF, Dekker HA, et al. Cytomegalovirus seroprevalence as a risk factor for poor outcome in acute respiratory distress syndrome*. Crit Care Med. 2015;43(2):394-400

28. Systematic Reviews: CRD's guidance for undertaking reviews in health care 2009;1:281. https://www.york.ac.uk/media/crd/Systematic_Reviews.pdf.

29. Sampson M, McGowan J, Cogo E, Grimshaw J, Moher D, Lefebvre C. An evidence-based practice guideline for the peer review of electronic search strategies. J Clin Epidemiol. 2009;62(9):944-52.

30. Wells GA SB, O'Connell D, et al. The Newcastle-Ottawa Scale (NOS) for assessing the quality of nonrandomised studies in meta-analyses Ottawa. ON: Ottawa: Hospital Research Institute; 2011. p. [cited 2016].

31. Higgins JP, Altman DG, Gotzsche PC, Juni P, Moher D, Oxman AD, et al. The Cochrane Collaboration's tool for assessing risk of bias in randomised trials. Bmj. 2011;343:d5928.

32. Higgins JP, Thompson SG. Quantifying heterogeneity in a meta-analysis. Stat Med. 2002;21(11):1539-58.

33. Egger M, Davey Smith G, Schneider M, Minder C. Bias in meta-analysis detected by a simple, graphical test. BMJ. 1997;315(7109):629-34.

34. Fryer JF, The collaborative study group, Heath ABAR, Minor PD. Collaborative study to evaluate the proposed 1st WHO International Standard for human cytomegalovirus (HCMV) for nucleic acid amplification (NAT)-based assays. 2010

\section{Submit your next manuscript to BioMed Central and we will help you at every step:}

- We accept pre-submission inquiries

- Our selector tool helps you to find the most relevant journal

- We provide round the clock customer support

- Convenient online submission

- Thorough peer review

- Inclusion in PubMed and all major indexing services

- Maximum visibility for your research

Submit your manuscript at www.biomedcentral.com/submit

C Biomed Central 